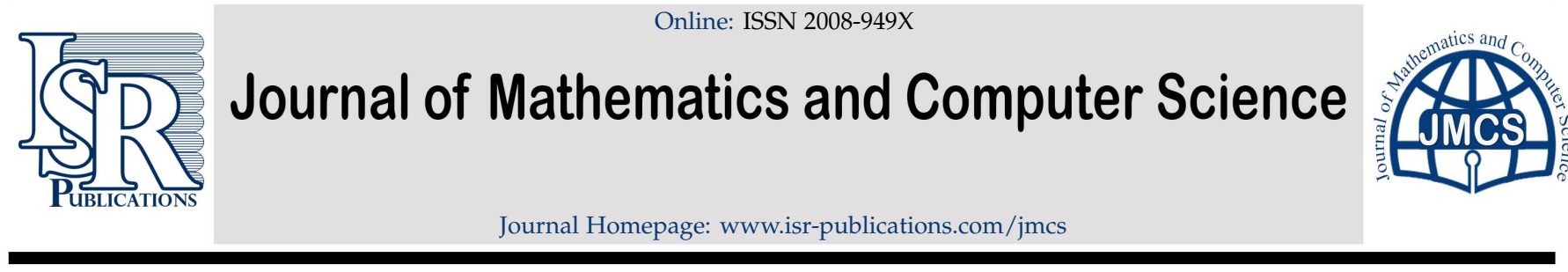

\title{
Essentiality and fixed point results for Eilenberg-Montgomery type maps
}

\author{
Donal O'Regan \\ School of Mathematics, Statistics and Applied Mathematics, National University of Ireland, Galway, Ireland.
}

\begin{abstract}
In this paper we establish topological transversality theorems so in particular general Leray-Schauder type alternatives and general Furi-Pera type results for Eilenberg-Montgomery type maps.
\end{abstract}

Keywords: Eilenberg-Montgomery maps, essential maps, Leray-Schauder alternatives, continuation theory.

2020 MSC: 47H10, 54C60, 54H25, 55M20.

(C)2021 All rights reserved.

\section{Introduction}

Coincidence theory in a general setting for acyclic maps was initiated by O'Regan in [9]. This paper is motivated by a fixed point result of Eilenberg and Montgomery [1] where they considered maps of the form $f F$ where $f$ is a continuous single valued map and $F$ is an acyclic map. In this paper we present a general continuation theory for Eilenberg-Montgomery type maps. Even though some of the results presented here could be modified from the results of $O^{\prime}$ Regan $[9,10]\left(\Phi\right.$ replaced by $f^{-1}$ there) however we feel it is more natural to construct this theory from a well known fixed point result. In particular we base our theory on a result of Gorniewicz [5, 8] since maps of Eilenberg-Montgomery type are admissible with respect to Gorniewicz. In this paper we present general Granas type topological transversality theorems $[6,7,10]$, general Leray-Schauder type alternatives [3, 7] and general Furi-Pera type results [4] for Eilenberg-Montgomery type maps.

Now we describe the maps considered in this paper. Let $\mathrm{H}$ be the C̆ech homology functor with compact carriers and coefficients in the field of rational numbers $\mathrm{K}$ from the category of Hausdorff topological spaces and continuous maps to the category of graded vector spaces and linear maps of degree zero. Thus $H(X)=\left\{H_{q}(X)\right\}$ (here $X$ is a Hausdorff topological space) is a graded vector space, $H_{q}(X)$ being the $q-$ dimensional Čech homology group with compact carriers of $X$. For a continuous map $f: X \rightarrow X, H(f)$ is the induced linear map $f_{\star}=\left\{f_{\star q}\right\}$, where $f_{\star q}: H_{q}(X) \rightarrow H_{q}(X)$. A space $X$ is acyclic if $X$ is nonempty, $\mathrm{H}_{\mathrm{q}}(X)=0$ for every $\mathrm{q} \geqslant 1$, and $\mathrm{H}_{0}(X) \approx \mathrm{K}$.

Let $X, Y$ and $\Gamma$ be Hausdorff topological spaces. A continuous single valued map $p: \Gamma \rightarrow X$ is called a Vietoris map (written $p: \Gamma \Rightarrow X$ ) if the following two conditions are satisfied:

Email address: donal . oregan@nuigalway.ie (Donal O'Regan)

doi: $10.22436 /$ jmcs.022.04.07

Received: 2020-06-11 Revised: 2020-07-15 Accepted: 2020-07-30 
(i) for each $x \in X$, the set $p^{-1}(x)$ is acyclic;

(ii) $p$ is a perfect map, i.e., $p$ is closed and for every $x \in X$ the set $p^{-1}(x)$ is nonempty and compact.

Let $\phi: X \rightarrow Y$ be a multi-valued map (note for each $x \in X$ we assume $\phi(x)$ is a nonempty subset of $Y)$. A pair $(p, q)$ of single valued continuous maps of the form $X \stackrel{p}{\leftarrow} \Gamma \stackrel{q}{\rightarrow} Y$ is called a selected pair of $\phi$ (written $(p, q) \subset \phi)$ if the following two conditions hold:

(i) $p$ is a Vietoris map;

(ii) $\mathrm{q}\left(\mathrm{p}^{-1}(\mathrm{x})\right) \subset \phi(\mathrm{x})$ for any $\mathrm{x} \in \mathrm{X}$.

Now we define the admissible maps of Gorniewicz [5]. A upper semicontinuous map $\phi: X \rightarrow Y$ with closed values is said to be admissible (and we write $\phi \in \operatorname{Ad}(X, Y)$ ) provided there exists a selected pair $(p, q)$ of $\phi$. An example of an admissible map is an acyclic map. A upper semicontinuous map $\phi: X \rightarrow K(Y)$ is said to be an acyclic map; here $\mathrm{K}(\mathrm{Y})$ denotes the family of nonempty, acyclic, compact subsets of $Y$.

By a space we mean a Hausdorff topological space. Let $Q$ be a class of topological spaces. A space $Y$ is an extension space for $Q$ (written $Y \in E S(Q)$ if for all $X \in Q$ and all $K \subseteq X$ closed in $X$, any continuous function $f_{0}: K \rightarrow Y$ extends to a continuous function $f: X \rightarrow Y$.

Now we recall the following fixed point result in the literature $[5,8]$.

Theorem 1.1. Let $X \in \mathrm{ES}($ compact $)$ and $\Psi \in \operatorname{Ad}(X, X)$ a compact map. Then there exists $a x \in X$ with $x \in \Psi(x)$.

\section{Continuation theory}

Let $E$ be a completely regular topological space and $U$ an open subset of $E$. In this section we fix a continuous single valued map $f: E \rightarrow E$.

Definition 2.1. We say $F \in A(\bar{U}, E)$ if $F: \bar{U} \rightarrow K(E)$ is a upper semi-continuous compact map; here $K(E)$ denotes the family of nonempty, compact, acyclic subsets of $E$ and $\bar{U}$ denotes the closure of $U$ in $E$.

Definition 2.2. We say $F \in A_{\partial u}(\bar{U}, E)$ if $F \in A(\bar{U}, E)$ with $x \notin f(F(x))$ for $x \in \partial U$; here $\partial U$ denotes the boundary of $\mathrm{U}$ in $\mathrm{E}$.

Definition 2.3. Let $F, G \in A_{\partial u}(\bar{U}, E)$. We say $F \cong G$ in $A_{\partial u}(\bar{U}, E)$ if there exists a upper semicontinuous compact map $H: \bar{U} \times[0,1] \rightarrow 2^{E}$ with $H_{t} \in A(\bar{U}, E)$ for each $t \in(0,1), x \notin f\left(H_{t}(x)\right)$ for $x \in \partial U$ and $\mathrm{t} \in(0,1)$ (here $\left.\mathrm{H}_{\mathrm{t}}(\mathrm{x})=\mathrm{H}(\mathrm{x}, \mathrm{t})\right), \mathrm{H}_{0}=\mathrm{F}$ and $\mathrm{H}_{1}=\mathrm{G}$.

Remark 2.4. Note that $\cong$ in $A_{\partial u}(\bar{U}, E)$ is an equivalence relation.

Next we present the notion of an EM-essential map.

Definition 2.5. We say $F \in A_{\partial u}(\bar{U}, E)$ is $E M$-essential in $A_{\partial u}(\bar{U}, E)$ if for any map $J \in A_{\partial u}(\bar{U}, E)$ with $\left.\mathrm{J}\right|_{\partial \mathrm{u}}=\left.\mathrm{F}\right|_{\partial \mathrm{u}}$ there exists a $\mathrm{x} \in \mathrm{U}$ with $\mathrm{x} \in \mathrm{f}(\mathrm{J}(\mathrm{x}))$.

Theorem 2.6. Let $\mathrm{E}$ be a completely regular topological space and $\mathrm{U}$ an open subset of $\mathrm{E}$. Let $\mathrm{F} \in \mathrm{A}_{\mathrm{\partial}} \mathrm{u}(\overline{\mathrm{U}}, \mathrm{E})$ and suppose $\mathrm{G} \in \mathrm{A}_{\partial \mathrm{u}}(\overline{\mathrm{U}}, \mathrm{E})$ is $\mathrm{EM}$-essential in $\mathrm{A}_{\partial \mathrm{u}}(\overline{\mathrm{U}}, \mathrm{E})$. Also suppose

$$
\text { for any map } \mathrm{J} \in \mathrm{A}_{\partial \mathrm{u}}(\overline{\mathrm{U}}, \mathrm{E}) \text { with }\left.\mathrm{J}\right|_{\partial \mathrm{u}}=\left.\mathrm{F}\right|_{\partial \mathrm{u}} \text {, we have } \mathrm{G} \cong \mathrm{J} \text { in } \mathrm{A}_{\partial \mathrm{u}}(\overline{\mathrm{U}}, \mathrm{E}) \text {. }
$$

Then $\mathrm{F}$ is EM-essential in $\mathrm{A}_{\partial \mathrm{u}}(\overline{\mathrm{U}}, \mathrm{E})$.

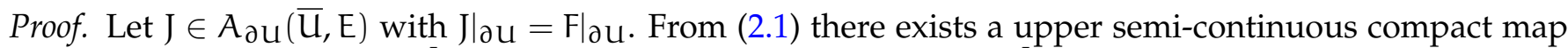
$H^{J}: \bar{U} \times[0,1] \rightarrow 2^{E}$ with $H_{t}^{J} \in A(\bar{U}, E)$ for each $t \in(0,1), x \notin f\left(H_{t}^{J}(x)\right)$ for $x \in \partial U$ and $t \in(0,1)$ (here $\left.H_{t}^{J}(x)=H^{J}(x, t)\right), H_{0}^{J}=G$ and $H_{1}^{J}=J$. Let

$$
K=\left\{x \in \bar{U}: x \in f\left(H_{t}^{J}(x)\right) \text { for some } t \in[0,1]\right\}, \quad \text { and } \quad D=\left\{(x, t) \in \bar{U} \times[0,1]: x \in f\left(H_{t}^{J}(x)\right)\right\} \text {. }
$$


Now $D \neq \emptyset$ (since $G$ is EM-essential in $A_{\partial u}(\bar{U}, E)$ ) and $D$ is closed (note $f$ is continuous and $H^{J}$ is upper semi-continuous) and so compact (note $\mathrm{H}^{\mathrm{J}}$ is a compact map). Let $\pi: \overline{\mathrm{U}} \times[0,1] \rightarrow \overline{\mathrm{U}}$ be the projection. Now $K=\pi(D)$ is closed (see Kuratowski's theorem [2]) and so in fact compact (recall projections are continuous). Also note $K \cap \partial U=\emptyset$ (since $x \notin f\left(H_{t}^{J}(x)\right)$ for $x \in \partial U$ and $t \in(0,1)$ ) so since $E$ is Tychonoff there exists a continuous map $\mu: \overline{\mathrm{U}} \rightarrow[0,1]$ with $\mu(\partial \mathrm{U})=0$ and $\mu(\mathrm{K})=1$. Let $\mathrm{R}(\mathrm{x})=\mathrm{H}^{\mathrm{J}}(\mathrm{x}, \mu(\mathrm{x}))=$ $H_{\mu(x)}^{J}(x)$ for $x \in \bar{U}$. Now $R \in A_{\partial u}(\bar{U}, E)$ with $\left.R\right|_{\partial u}=\left.G\right|_{\partial u}$ (note if $x \in \partial U$ then $R(x)=H^{J}(x, 0)=G(x)$ ). Now since $G$ is $E M$-essential in $A_{\partial u}(\bar{U}, E)$ there exists a $x \in U$ with $x \in f(R(x))$, i.e., $x \in f\left(H_{\mu(x)}^{J}(x)\right)$. Thus $x \in K$ so $\mu(x)=1$ and as a result $x \in f\left(H_{1}^{J}(x)\right)=f(J(x))$ and we are finished.

We now present the topological transversality theorem for $A_{\partial u}(\bar{U}, E)$ maps. To do this we need an extra assumption (which will be discussed after the proof of our next result):

$$
\text { if } F, G \in A_{\partial u}(\bar{U}, E) \text { with }\left.F\right|_{\partial u}=\left.G\right|_{\partial u} \text {, then } F \cong G \text { in } A_{\partial u}(\bar{U}, E) \text {. }
$$

Theorem 2.7. Let $\mathrm{E}$ be a completely regular topological space and $\mathrm{U}$ an open subset of $\mathrm{E}$. Suppose (2.2) holds. Let $\mathrm{F}$ and $\mathrm{G}$ be two maps in $\mathrm{A}_{\partial \mathrm{u}}(\overline{\mathrm{U}}, \mathrm{E})$ with $\mathrm{F} \cong \mathrm{G}$ in $\mathrm{A}_{\partial \mathrm{u}}(\overline{\mathrm{U}}, \mathrm{E})$. Now $\mathrm{F}$ is $\mathrm{EM}$-essential in $\mathrm{A}_{\partial \mathrm{u}}(\overline{\mathrm{U}}, \mathrm{E})$ if and only if $\mathrm{G}$ is $\mathrm{EM}$-essential in $\mathrm{A}_{\partial \mathrm{u}}(\overline{\mathrm{U}}, \mathrm{E})$.

Proof. Assume $G$ is $E M$-essential in $A_{\partial u}(\bar{U}, E)$. Let $J \in A_{\partial u}(\bar{U}, E)$ with $\left.J\right|_{\partial u}=\left.F\right|_{\partial u}$. From (2.2) we have $J \cong F$ in $A_{\partial u}(\bar{U}, E)$ and since $F \cong G$ in $A_{\partial u}(\bar{U}, E)$ we have $G \cong J$ in $A_{\partial u}(\bar{U}, E)$, i.e., (2.1) holds. Now Theorem 2.6 guarantees that $F$ is $E M$-essential in $A_{\partial u}(\bar{U}, E)$. Similarly if $F$ is EM-essential in $A_{\partial u}(\bar{U}, E)$ then $G$ is $E M$-essential in $A_{\partial u}(\bar{U}, E)$.

Now we discuss (2.2). Let $E$ be a topological (Hausdorff) vector space and $U$ an open convex subset of E. Suppose

$$
\text { there exists a retraction } r: \overline{\mathrm{U}} \rightarrow \partial \mathrm{u} \text {, }
$$

(note (2.3) holds if $E$ is an infinite dimensional Banach space). We now show that (2.2) holds. To see this let $r$ be as in (2.3) and $F, G \in A_{\partial u}(\bar{U}, E)$ with $\left.F\right|_{\partial u}=\left.G\right|_{\partial u}$. Let $F^{\star}(x)=F(r(x))$ for $x \in \bar{U}$. Note $\mathrm{F}^{\star}(\mathrm{x})=\mathrm{G}(\mathrm{r}(\mathrm{x})), \mathrm{x} \in \overline{\mathrm{U}}$ since $\left.\mathrm{F}\right|_{\partial \mathrm{u}}=\left.\mathrm{G}\right|_{\partial \mathrm{u}}$. Take

$$
\Lambda(x, \lambda)=\mathrm{G}(2 \lambda \mathrm{r}(x)+(1-2 \lambda) x)=\mathrm{G} \circ \mathrm{j}(x, \lambda) \text { for }(x, \lambda) \in \overline{\mathrm{U}} \times\left[0, \frac{1}{2}\right] ;
$$

here $j: \bar{U} \times\left[0, \frac{1}{2}\right] \rightarrow \bar{U}$ (note $\bar{U}$ is convex) is given by $j(x, \lambda)=2 \lambda r(x)+(1-2 \lambda) x$. Notice $G \cong F^{\star}$ in $A_{\partial u}(\bar{U}, E)$; note $\Lambda: \bar{U} \times\left[0, \frac{1}{2}\right] \rightarrow 2^{E}$ is a upper semi-continuous compact map and for fixed $x \in \bar{U}$ and $t \in\left[0, \frac{1}{2}\right]$, note $\Lambda_{t}(x)$ has acyclic values and note $x \notin f\left(\Lambda_{t}(x)\right)$ for $x \in \partial U$ and $t \in\left[0, \frac{1}{2}\right]$ since if $x \in \partial U$ and $t \in\left[0, \frac{1}{2}\right]$, then $r(x)=x$ so $\Lambda_{t}(x)=G(x)$ and $f\left(\Lambda_{t}(x)\right)=f(G(x))$. Similarly if $\Theta(x, \lambda)=$ $\mathrm{F}((2-2 \lambda) r(x)+(2 \lambda-1) x)$ for $(x, \lambda) \in \overline{\mathrm{U}} \times\left[\frac{1}{2}, 1\right]$, then $\mathrm{F}^{\star} \cong \mathrm{F}$ in $A_{\partial \mathrm{u}}(\overline{\mathrm{U}}, \mathrm{E})$. Thus (2.2) holds.

To establish Leray-Schauder type alternatives first we present an example of a EM-essential in $A_{\partial u}(\bar{U}, E)$ map.

Theorem 2.8. Let $\mathrm{E}$ be a locally convex metrizable topological vector space, $\mathrm{U}$ an open subset of $\mathrm{E}$, and $\mathrm{f}(0) \in \mathrm{U}$. Then the zero map is $\mathrm{EM}$-essential in $\mathrm{A}_{\partial \mathrm{u}}(\overline{\mathrm{U}}, \mathrm{E})$.

Proof. Let $\mathrm{G} \in \mathrm{A}_{\partial \mathrm{u}}(\overline{\mathrm{U}}, \mathrm{E})$ with $\left.\mathrm{J}\right|_{\partial \mathrm{u}}=\left.\{0\}\right|_{\partial \mathrm{u}}$. We must show there exists a $x \in \mathrm{U}$ with $x \in f(G(x))$. Let

$$
\Psi(x)= \begin{cases}G(x), & x \in \bar{U} \\ \{0\}, & x \in E \backslash \bar{U}\end{cases}
$$

Now $\Psi \in A(E, E)$ (a map $\theta \in A(E, E)$ if $\theta: E \rightarrow K(E)$ is a upper semi-continuous compact map) and so $\mathrm{f} \Psi$ is an admissible compact map. Now Theorem 1.1 (note every locally convex metrizable topological vector space is an AR) guarantees that there exists a $x \in E$ with $x \in f(\Psi(x))$. If $x \in E \backslash U$, then $x=f(0)$, a contradiction since $f(0) \in U$. Thus $x \in U$ so $x \in f(G(x))$. 
Remark 2.9. Let $E$ be a locally convex metrizable topological vector space, $U$ an open subset of $E, f(0) \in U$, $F \in A_{\partial U}(\bar{U}, E)$ and $x \notin f(t F(x))$ for $x \in \partial U$ and $t \in(0,1)$. Then one homotopy in $A_{\partial u}(\bar{U}, E)$ from $F$ to 0 (i.e., so $0 \cong F$ in $\left.A_{\partial u}(\bar{U}, E)\right)$ is $H(x, t)=t F(x)$ for $t \in[0,1]$ and $x \in \bar{U}$. To see this note $H: \bar{U} \times[0,1] \rightarrow 2^{E}$ is a upper semi-continuous compact map and note for a fixed $t \in[0,1]$ and a fixed $x \in \bar{U}$, then $H_{t}(x)$ is acyclic valued (recall homeomorphic spaces have isomorphic homology groups) so $H_{t} \in A_{\partial u}(\bar{U}, E)$. Finally $\mathrm{H}_{0}=0$ and $\mathrm{H}_{1}=\mathrm{F}$ so $0 \cong \mathrm{F}$ in $\mathrm{A}_{\partial \mathrm{u}}(\overline{\mathrm{U}}, \mathrm{E})$.

Theorem 2.10. Let $\mathrm{E}$ be a locally convex metrizable topological vector space, $\mathrm{U}$ an open subset of $\mathrm{E}, \mathrm{F} \in \mathrm{A}_{\partial \mathrm{u}}(\overline{\mathrm{U}}, \mathrm{E})$, $\mathrm{f}(0) \in \mathrm{U}$ and $\mathrm{x} \notin \mathrm{f}(\mathrm{tF}(\mathrm{x}))$ for $\mathrm{x} \in \partial \mathrm{U}$ and $\mathrm{t} \in(0,1)$. Then $\mathrm{F}$ is $\mathrm{EM}$-essential in $\mathrm{A}_{\partial \mathrm{u}}(\overline{\mathrm{U}}, \mathrm{E})$ (so in particular there exists a $\mathrm{x} \in \mathrm{U}$ with $\mathrm{x} \in \mathrm{f}(\mathrm{F}(\mathrm{x}))$ ).

Proof. From Theorem 2.8 we know that the zero map is EM-essential in $A_{\partial u}(\bar{U}, E)$. We will apply Theorem 2.6 to show $F$ is $E M$-essential in $A_{\partial u}(\bar{U}, E)$. Note topological vector spaces are completely regular so we need only to show (2.1) holds with $G=0$. Consider any map $J \in A_{\partial u}(\bar{U}, E)$ with $\left.J\right|_{\partial u}=\left.F\right|_{\partial u}$. Now let $H^{J}(x, t)=t J(x)$ and similar to Remark 2.9 note $J \cong 0$ in $A_{\partial u}(\bar{U}, E)$ (note if $x \in \partial U$ and $t \in(0,1)$, then since $\left.J\right|_{\text {au }}=\left.F\right|_{\text {au }}$ we have $\left.f(t J(x))=f(t F(x))\right)$. Thus (2.1) holds.

Remark 2.11. Theorem 2.10 gives a strong conclusion, namely $F$ is EM-essential in $A_{\partial u}(\bar{U}, E)$. The usual conclusion in a Leray-Schauder type alternative is that there exists a $x \in U$ with $x \in f(F(x))$. We note that this can be proved directly without any reference to essential maps. Let

$$
\mathrm{K}=\{x \in \overline{\mathrm{U}}: x \in \mathrm{f}(\mathrm{tF}(\mathrm{x})) \text { for some } \mathrm{t} \in[0,1]\} \text {. }
$$

Note $K \neq \emptyset$ (take $t=0$ and $x=f(0)$ ) is compact and $K \cap \partial U=\emptyset$ (since $x \notin f(t F(x)$ ) for $x \in \partial U$ and $\mathrm{t} \in(0,1))$ so there exists a continuous map $\mu: \overline{\mathrm{U}} \rightarrow[0,1]$ with $\mu(\partial \mathrm{U})=0$ and $\mu(\mathrm{K})=1$. Let $\mathrm{N}: \mathrm{E} \rightarrow 2^{\mathrm{E}}$ be given by

$$
N(x)= \begin{cases}\mu(x) F(x), & x \in \bar{U}, \\ \{0\}, & x \in E \backslash \bar{U} .\end{cases}
$$

Now $N \in A(E, E)$ so $f N$ is an admissible compact map. Then Theorem 1.1 guarantees that there exists a $x \in E$ with $x \in f(N(x))$. If $x \in E \backslash U$, then $x=f(0)$, a contradiction since $f(0) \in U$. Thus $x \in U$ so $x \in f(\mu(x) F(x))$ and as a result $x \in K$. Thus $\mu(x)=1$ and so $x \in f(F(x))$.

Now we prove a Furi-Pera type result. Here $E$ will be a locally convex metrizable topological vector space and $\mathrm{Q}$ a closed convex subset of $\mathrm{E}$. In our next result we assume $\partial \mathrm{Q}=\mathrm{Q}$ (the case when $\operatorname{int}(\mathrm{Q}) \neq \emptyset$ is also easily handled; see Remark 2.13).

Theorem 2.12. Let $\mathrm{E}$ be a locally convex metrizable topological vector space, $\mathrm{Q}$ a closed convex subset of $\mathrm{E}, \partial \mathrm{Q}=\mathrm{Q}$, $\mathrm{F} \in \mathrm{A}(\mathrm{Q}, \mathrm{E})$ (i.e., $\mathrm{F}: \mathrm{Q} \rightarrow \mathrm{K}(\mathrm{E})$ a upper semi-continuous, compact map) and $\mathrm{f}: \mathrm{E} \rightarrow \mathrm{E}$ a continuous single valued map with $\mathrm{f}(0) \in \mathrm{Q}$. In addition assume

$$
\left\{\begin{array}{l}
\text { if }\left\{\left(x_{j}, \lambda_{j}\right)\right\}_{j=1}^{\infty} \text { is a sequence in } \partial \mathrm{Q} \times[0,1] \text { convergingto }(x, \lambda) \text { with } x \in f(\lambda \mathrm{F}(\mathrm{x})) \text { and } 0 \leqslant \lambda<1, \\
\text { then }\left\{\mathrm{f}\left(\lambda_{\mathrm{j}} \mathrm{F}\left(\mathrm{x}_{\mathrm{j}}\right)\right)\right\} \subseteq \mathrm{Q} \text { for } \mathrm{j} \text { sufficiently large. }
\end{array}\right.
$$

Then there exists $a x \in Q$ with $x \in f(F(x))$.

Proof. From Dugundji's theorem we know there exists a retraction $r: E \rightarrow Q$. Let

$$
\Omega=\{x \in E: x \in f(F(r(x))\}
$$

Note $\Omega \neq \emptyset$ from Theorem 1.1 (note fFr is a compact admissible map) and $\Omega$ is compact. We claim $\Omega \cap Q \neq \emptyset$. To show this we argue by contradiction. Suppose $\Omega \cap Q=\emptyset$. Then since $\Omega$ is compact and $Q$ is closed there exists a $\delta>0$ with $\operatorname{dist}(Q, \Omega)>\delta$. Choose $m \in\{1,2, \ldots\}$ with $1<\delta m$ and let

$$
\mathrm{U}_{i}=\left\{x \in E: d(x, Q)<\frac{1}{i}\right\} \text { for } i \in\{m, m+1, \ldots\}
$$


here $d$ is the metric associated with E. Fix $i \in\{m, m+1, \ldots\}$. Since $\operatorname{dist}(Q, \Omega)>\delta$ we see that $\Omega \cap \overline{U_{i}}=\emptyset$. Now Remark 2.11 (note $\mathrm{Fr}$ has acyclic values so $\mathrm{Fr}$ is a compact acyclic map and $f(0) \in \mathrm{Q} \subseteq \mathrm{U}_{i}$ ) guarantees that there exists $\lambda_{i} \in(0,1)$ and $y_{i} \in \partial u_{i}$ with $y_{i} \in f\left(\lambda_{i} \operatorname{Fr}\left(y_{i}\right)\right)$. Since $y_{i} \in \partial u_{i}$ we have

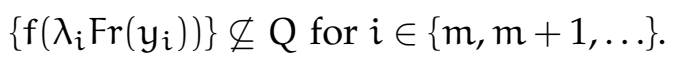

Let

$$
\mathrm{D}=\{x \in \mathrm{E}: x \in \mathrm{f}(\lambda \operatorname{Fr}(\mathrm{x})) \text { for some } \lambda \in[0,1]\} .
$$

Now $\mathrm{D} \neq \emptyset$ (see Theorem 1.1 and take $\lambda=1$ ) and $\mathrm{D}$ is compact. This together with

$$
d\left(y_{j}, Q\right)=\frac{1}{j} \text { and }\left|j_{j}\right| \leqslant 1 \text { for } j \in\{m, m+1, \ldots\}
$$

implies that we may assume without loss of generality that $\lambda_{j} \rightarrow \lambda^{\star} \in[0,1]$ and $y_{j} \rightarrow y^{\star} \in \partial Q$. In addition since $f$ and $r$ are continuous, $F$ is upper semicontinuous and $y_{j} \in f\left(\lambda_{j} \operatorname{Fr}\left(y_{j}\right)\right)$, we have $y^{\star} \in f\left(\lambda^{\star} \operatorname{Fr}\left(y^{\star}\right)\right)$. Thus, since $r\left(y^{\star}\right)=y^{\star}$, we have $y^{\star} \in f\left(\lambda^{\star} F y^{\star}\right)$. If $\lambda^{\star}=1$, then $y^{\star} \in f\left(F y^{\star}\right)\left(=f\left(F r\left(y^{\star}\right)\right)\right.$, which contradicts $\Omega \cap Q=\emptyset$. Thus $0 \leqslant \lambda^{\star}<1$. Now (2.4) with $x_{j}=r\left(y_{j}\right)$ (note $y_{j} \in \partial u_{j}$ and $r\left(y_{j}\right) \in \partial Q$ ) and $x=y^{\star}=r\left(y^{\star}\right)$ and $y^{\star} \in f\left(\lambda^{\star} F\left(y^{\star}\right)\right)$ implies

$$
\left\{f\left(\lambda_{j} F x_{j}\right)\right\} \subseteq Q \text { for } j \text { sufficiently large. }
$$

This contradicts (2.5). Thus $\Omega \cap Q \neq \emptyset$ so there exists a $x \in Q$ with $x \in f(\operatorname{Fr}(x))=f(F x)$.

Remark 2.13. In Theorem 2.12 we assumed $\partial \mathrm{Q}=\mathrm{Q}$. However this is easily removed since if $\operatorname{int}(\mathrm{Q}) \neq \emptyset$ (assume without loss of generality that $0 \in \operatorname{int}(Q)$ ), then one can take the retraction $r: E \rightarrow Q$ as

$$
r(x)=\frac{x}{\max \{1, \mu(x)\}} \text { for } x \in E,
$$

where $\mu$ is the Minkowski functional on $Q$ (i.e., $\mu(x)=\inf \{\alpha>0: x \in \alpha Q\}$. Note $r(z) \in \partial Q$ if $z \in E \backslash Q$. The argument in Theorem 2.12 now remains the same.

However when $0 \in \operatorname{int}(Q)$, condition (2.4) can be linked to $x \notin f(\lambda F(x))$ for $x \in \partial U$ and $\lambda \in(0,1)$ in Theorem 2.10 (Remark 2.11); here $U=\operatorname{int}(Q)$. For simplicity take $f=i$ (identity), $U=\operatorname{int} Q$ and let $\mathrm{F}: \overline{\mathrm{U}} \rightarrow \mathrm{E}$ be a continuous single valued compact map with $x \neq F x$ for $x \in \partial \mathrm{U}$. Now we claim if (2.4) holds (i.e., if $\left\{\left(x_{j}, \lambda_{j}\right)\right\}_{j=1}^{\infty}$ is a sequence in $\partial Q \times[0,1]$ converging to $(x, \lambda)$ with $x=\lambda F x$ and $0 \leqslant \lambda<1$, then $\lambda_{j} F\left(x_{j}\right) \in Q$ for $j$ sufficiently large), then if $x \in \partial U$ and $\lambda \in(0,1)$, then $x \neq \lambda F x$. Suppose the claim is false. Then there exists a $x \in \partial U$ and $\lambda \in(0,1)$ with $x=\lambda F x$. Let $\left\{\left(x_{j}, \lambda_{j}\right)\right\}_{j=1}^{\infty}$ be a sequence in $\partial Q \times[0,1]$ with $x_{j}=x, \lambda_{j} \rightarrow \lambda$ and $\lambda_{j}>\lambda$. Now (2.4) implies that $\lambda_{j} F x \in Q$ for $j$ sufficiently large. However

$$
\mu\left(\lambda_{j} F x\right)=\mu\left(\frac{\lambda_{j}}{\lambda} \lambda F x\right)=\mu\left(\frac{\lambda_{j}}{\lambda} x\right)=\frac{\lambda_{j}}{\lambda} \mu(x)=\frac{\lambda_{j}}{\lambda}>1
$$

so $\lambda_{j} F x \notin Q$, a contradiction. Thus $x \neq \lambda F x$ for $x \in \partial U$ and $\lambda \in(0,1)$. Theorem 2.10 guarantees that $F$ has a fixed point in $\mathrm{U}$.

Remark 2.14. Note one can choose $d$ to be a translational invariant metric associated with $E$ so each $U_{i}$ $(i \in\{1,2, \ldots\})$ in the proof of Theorem 2.12 could be convex.

To take (2.2) into account in the topological transversality theorem one could replace the definition of EM-essential in $A_{\partial u}(\bar{U}, E)$ with the following definition.

Definition 2.15. We say $F \in A_{\partial u}(\bar{U}, E)$ is EM-essential in $A_{\partial u}(\bar{U}, E)$ if for any map $J \in A_{\partial u}(\bar{U}, E)$ with $\left.\mathrm{J}\right|_{\partial \mathrm{u}}=\left.\mathrm{F}\right|_{\partial \mathrm{u}}$ and $\mathrm{J} \cong \mathrm{F}$ in $A_{\partial \mathrm{u}}(\overline{\mathrm{U}}, \mathrm{E})$ there exists a $\mathrm{x} \in \mathrm{U}$ with $\mathrm{x} \in \mathrm{f}(\mathrm{J}(\mathrm{x}))$. 
Theorem 2.16. Let $\mathrm{E}$ be a completely regular topological space and $\mathrm{U}$ an open subset of $\mathrm{E}$. Let $\mathrm{F} \in \mathrm{A}_{\mathrm{\partial u}}(\overline{\mathrm{U}}, \mathrm{E})$ and suppose $\mathrm{G} \in \mathrm{A}_{\partial \mathrm{u}}(\overline{\mathrm{U}}, \mathrm{E})$ is $\mathrm{EM}$-essential in $\mathrm{A}_{\partial \mathrm{u}}(\overline{\mathrm{U}}, \mathrm{E})$ (Definition 2.15). Also suppose

for any map $\mathrm{J} \in \mathrm{A}_{\partial \mathrm{u}}(\overline{\mathrm{U}}, \mathrm{E})$ with $\left.\mathrm{J}\right|_{\partial \mathrm{u}}=\left.\mathrm{F}\right|_{\partial \mathrm{u}}$, and $\mathrm{J} \cong \mathrm{F}$ in $\mathrm{A}_{\partial \mathrm{u}}(\overline{\mathrm{U}}, \mathrm{E})$, we have $\mathrm{G} \cong \mathrm{J}$, in $\mathrm{A}_{\partial \mathrm{u}}(\overline{\mathrm{U}}, \mathrm{E})$.

Then $\mathrm{F}$ is $\mathrm{EM}$-essential in $\mathrm{A}_{\partial \mathrm{u}}(\overline{\mathrm{U}}, \mathrm{E})$ (Definition 2.15).

Proof. Let $\mathrm{J} \in \mathrm{A}_{\partial \mathrm{u}}(\overline{\mathrm{U}}, \mathrm{E})$ with $\left.\mathrm{J}\right|_{\partial \mathrm{u}}=\left.\mathrm{F}\right|_{\partial u}$ and $\mathrm{J} \cong \mathrm{F}$ in $A_{\partial \mathrm{u}}(\overline{\mathrm{U}}, \mathrm{E})$. From (2.1) there exists a upper semicontinuous compact map $H^{J}: \bar{U} \times[0,1] \rightarrow 2^{E}$ with $H_{t}^{J} \in A(\bar{U}, E)$ for each $t \in(0,1), x \notin f\left(H_{t}^{J}(x)\right)$ for $x \in \partial U$ and $t \in(0,1)$ (here $\left.H_{t}^{J}(x)=H^{J}(x, t)\right), H_{0}^{J}=G$ and $H_{1}^{J}=J$. Let

$$
K=\left\{x \in \bar{U}: x \in f\left(H_{t}^{J}(x)\right) \text { for some } t \in[0,1]\right\} .
$$

Now $\mathrm{K} \neq \emptyset$ is compact, $\mathrm{K} \cap \partial \mathrm{U}=\emptyset$ so there exists a continuous map $\mu: \overline{\mathrm{U}} \rightarrow[0,1]$ with $\mu(\partial \mathrm{U})=0$ and $\mu(K)=1$. Let $R(x)=H_{\mu(x)}^{J}(x)$ for $x \in \bar{U}$. Now $R \in A_{\partial u}(\bar{U}, E)$ with $\left.R\right|_{\partial u}=\left.G\right|_{\partial u}$. We now claim $G \cong R$ in $A_{\partial u}(\bar{U}, E)$. If the claim is true, then since $G$ is EM-essential in $A_{\partial u}(\bar{U}, E)$ (Definition 2.15) there exists a $x \in U$ with $x \in f(R(x))$, i.e., $x \in f\left(H_{\mu(x)}^{J}(x)\right)$. Thus $x \in K$ so $\mu(x)=1$ and as a result $x \in f\left(H_{1}^{J}(x)\right)=f(J(x))$ and we are finished. It remains to prove the claim. To see this let $\mathrm{Q}: \overline{\mathrm{U}} \times[0,1] \rightarrow 2^{\mathrm{E}}$ be given by $\mathrm{Q}(\mathrm{x}, \mathrm{t})=\mathrm{H}^{\mathrm{J}}(\mathrm{x}, \mathrm{t} \mu(\mathrm{x}))=\mathrm{H}_{\mathrm{t} \mu(\mathrm{x})}^{\mathrm{J}}(\mathrm{x})$ and note $\mathrm{Q}: \overline{\mathrm{U}} \times[0,1] \rightarrow 2^{\mathrm{E}}$ is a upper semicontinuous compact map with $Q_{s}=H_{s \mu(.)}^{J} \in A(\bar{U}, E)$ for each $s \in[0,1]$ and $x \notin f\left(Q_{t}(x)\right)$ for $x \in \partial U$ and $t \in(0,1)$ (note if $x \in \partial U$ and $t \in(0,1)$, then $Q_{t}(x)=H_{t \mu(x)}^{J}(x)=H_{t}^{J}(x)$ since $\left.x \in K\right)$.

Theorem 2.17. Let $\mathrm{E}$ be a completely regular topological space and $\mathrm{U}$ an open subset of $\mathrm{E}$. Let $\mathrm{F}$ and $\mathrm{G}$ be two maps in $\mathrm{A}_{\partial \mathrm{u}}(\overline{\mathrm{U}}, \mathrm{E})$ with $\mathrm{F} \cong \mathrm{G}$ in $\mathrm{A}_{\partial \mathrm{u}}(\overline{\mathrm{U}}, \mathrm{E})$. Now $\mathrm{F}$ is EM-essential in $\mathrm{A}_{\partial \mathrm{u}}(\overline{\mathrm{U}}, \mathrm{E})$ (Definition 2.15) if and only if $\mathrm{G}$ is $\mathrm{EM}$-essential in $\mathrm{A}_{\partial \mathrm{u}}(\overline{\mathrm{U}}, \mathrm{E})$ (Definition 2.15).

Proof. Assume $G$ is $E M$-essential in $A_{\partial u}(\bar{U}, E)$ (Definition 2.15). Let $J \in A_{\partial u}(\bar{U}, E)$ with $\left.J\right|_{\partial u}=\left.F\right|_{\partial u}$ and $J \cong F$ in $A_{\partial u}(\bar{U}, E)$. Now since $F \cong G$ in $A_{\partial u}(\bar{U}, E)$ we have $G \cong J$ in $A_{\partial u}(\bar{U}, E)$, i.e., (2.6) holds. Now Theorem 2.16 guarantees that $F$ is EM-essential in $A_{\partial u}(\bar{U}, E)$ (Definition 2.15). Similarly if $F$ is EM-essential in $A_{\partial u}(\bar{U}, E)$ (Definition 2.15), then $G$ is EM-essential in $A_{\partial u}(\bar{U}, E)$ (Definition 2.15).

Now we present a general Leray-Schauder type result.

Theorem 2.18. Let $\mathrm{E}$ be a completely regular topological space, $\mathrm{U}$ an open subset of $\mathrm{E}, \mathrm{u}_{0} \in \mathrm{E}$ with $\mathrm{f}\left(\mathrm{u}_{0}\right) \in \mathrm{U}$, and $\mathrm{F} \in \mathrm{A}_{\mathrm{\partial u}}(\overline{\mathrm{U}}, \mathrm{E})$. Suppose the following:

$$
\text { for any } \Phi \in \mathrm{A}(\mathrm{E}, \mathrm{E}) \text { with } \Phi \cong\left\{\mathrm{u}_{0}\right\} \text { in } \mathrm{A}(\mathrm{E}, \mathrm{E}) \text {, there exists a } z \in \mathrm{E} \text { with } z \in \mathrm{f}(\Phi(z)) \text {. }
$$

Finally suppose $\mathrm{F} \cong\left\{\mathrm{u}_{0}\right\}$ in $\mathrm{A}_{\partial \mathrm{u}}(\overline{\mathrm{U}}, \mathrm{E})$. Then $\mathrm{F}$ is $\mathrm{EM}$-essential in $\mathrm{A}_{\partial \mathrm{u}}(\overline{\mathrm{U}}, \mathrm{E})$ (Definition 2.15).

Remark 2.19. We say $\Phi \cong\left\{u_{0}\right\}$ in $A(E, E)$ if there exists a upper semicontinuous, compact map $R: E \times$ $[0,1] \rightarrow 2^{E}$ with $R_{t} \in A(E, E)$ for each $t \in[0,1], R_{0}=\Phi$ and $R_{1}=\left\{u_{0}\right\}$.

Proof. Let $J(x)=\left\{u_{0}\right\}$ for $x \in E$. We show $J$ is EM-essential in $A_{\partial u}(\bar{U}, E)$ (Definition 2.15) so, then $F$ is $E M-e s s e n t i a l$ in $A_{\partial u}(\bar{U}, E)$ (Definition 2.15) from Theorem 2.17. Let $G \in A_{\partial u}(\bar{U}, E)$ with $\left.G\right|_{\partial u}=\left.J\right|_{\partial u}$ and $G \cong J$ in $A_{\partial u}(\bar{U}, E)$. We must show there exists a $x \in U$ with $x \in f(G(x))$. Since $G \cong J$ in $A_{\partial u}(\bar{U}, E)$ there exists a upper semicontinuous, compact map $\Psi: \overline{\mathrm{U}} \times[0,1] \rightarrow 2^{\mathrm{E}}$ with $\Psi_{\mathrm{t}} \in \mathrm{A}_{\text {วu }}(\overline{\mathrm{U}}, \mathrm{E})$ for each $\mathrm{t} \in[0,1]$, $\Psi_{0}=\mathrm{J}$ and $\Psi_{1}=\mathrm{G}$. Let

$$
\Omega=\left\{x \in \overline{\mathrm{U}}: x \in \mathrm{f}\left(\Psi_{\mathrm{t}}(\mathrm{x})\right) \text { for some } \mathrm{t} \in[0,1]\right\} .
$$

Note $\Omega \neq \emptyset$ (take $t=0$ and $x=f\left(u_{0}\right)$ ) is compact and $\Omega \cap(E \backslash U)=\emptyset$ (note $\Psi_{t} \in A_{\partial u}(\bar{U}, E)$ for $t \in[0,1]$ so $x \notin f\left(\Psi_{t}(x)\right)$ for $x \in \partial U$ and $\left.t \in(0,1)\right)$. Now there exists a continuous map $\sigma: E \rightarrow[0,1]$ with $\sigma(\Omega)=1$ and $\sigma(E \backslash U)=0$. Define $\Theta: E \times[0,1] \rightarrow 2^{E}$ by

$$
\Theta(x, t)= \begin{cases}\Psi(x, t \sigma(x)), & x \in \bar{U} \\ \left\{u_{0}\right\}, & x \in E \backslash U\end{cases}
$$


Note $\Theta: E \times[0,1] \rightarrow 2^{E}$ is an upper semicontinuous, compact map with $\Theta_{t} \in A(E, E)$ for each $t \in[0,1]$, so as a result $\Theta_{1} \cong \Theta_{0}=J$ in $A(E, E)$. Now (2.7) guarantees that there exists a $x \in E$ with $x \in f\left(\Theta_{1}(x)\right)$. If $x \in E \backslash \bar{U}$, then $x=f\left(u_{0}\right)$, a contradiction since $f\left(u_{0}\right) \in U$. Consequently $x \in U$ so $x \in f(\Psi(x, \sigma(x)))=$ $f\left(\Psi_{\sigma(x)}(x)\right)$ and as a result $x \in \Omega$ which implies $\sigma(x)=1$ and so $x \in f\left(\Psi_{1}(x)\right)=f(G(x))$.

Theorem 2.20. Let $\mathrm{E}$ be a (metrizable) ANR, $\mathrm{U}$ an open subset of $\mathrm{E}, \mathrm{u}_{0} \in \mathrm{E}$ with $\mathrm{f}\left(\mathrm{u}_{0}\right) \in \mathrm{U}, \mathrm{F} \in \mathrm{A}_{\partial \mathrm{u}}(\overline{\mathrm{U}}, \mathrm{E})$ and suppose $\mathrm{F} \cong\left\{\mathrm{u}_{0}\right\}$ in $\mathrm{A}_{\partial \mathrm{u}}(\overline{\mathrm{U}}, \mathrm{E})$. Then $\mathrm{F}$ is $\mathrm{EM}$-essential in $\mathrm{A}_{\partial \mathrm{u}}(\overline{\mathrm{U}}, \mathrm{E})$ (Definition 2.15).

Proof. The result follows from Theorem 2.18 once we show (2.7). Let $\Phi \in A(E, E)$ with $\Phi \cong\left\{u_{0}\right\}$ in $A(E, E)$. Then there exists a upper semicontinuous, compact map $R: E \times[0,1] \rightarrow 2^{E}$ with $R_{t} \in A(E, E)$ for each $t \in[0,1], R_{1}=\Phi$ and $R_{0}=\left\{u_{0}\right\}$. Note $E$ can be regarded as a closed subset of a normed space $X$ (see the Arens-Eells theorem). Since $E \in A N R$ there is an open neighborhood $V$ of $E$ in $X$ and a retraction (continuous) $r: \bar{V} \rightarrow E$. Let $\lambda: X \rightarrow[0,1]$ be a continuous function with $\lambda(X \backslash V)=0$ and $\lambda(E)=1$ and let

$$
Q(x)= \begin{cases}R(r(x), \lambda(x)), & x \in \bar{V} \\ \left\{u_{0}\right\}, & x \in X \backslash V .\end{cases}
$$

(note if $x \in \partial V$, then $Q(x)=R(r(x), 0)=R_{0}(r(x))=\left\{u_{0}\right\}$ ). Also note $Q \rightarrow 2^{X}$ is a upper semi-continuous, compact map and for fixed $x \in X$ note $Q(x)$ is acyclic valued, so $Q \in A(X, X)$. Thus $f Q$ is an admissible compact map so Theorem 1.1 guarantees that there exists a $x_{0} \in X$ with $x_{0} \in f\left(Q\left(x_{0}\right)\right)$. If $x_{0} \in X \backslash V$, then $x_{0}=f\left(u_{0}\right)$, a contradiction since $f\left(u_{0}\right) \in U \subseteq E \subseteq V$. If $x_{0} \in \bar{V} \backslash E$, then since $Q: X \rightarrow 2^{E}$ (note $\left.R: E \times[0,1] \rightarrow 2^{E}\right)$ and since $x_{0} \in f\left(Q\left(x_{0}\right)\right)$ one has $x_{0} \in E$, a contradiction. Thus $x_{0} \in E$ and so $r\left(x_{0}\right)=x_{0}$, $\lambda\left(x_{0}\right)=1$ and as a result $x_{0} \in f\left(R\left(x_{0}, 1\right)\right)=f\left(\Phi\left(x_{0}\right)\right)$, i.e., (2.7) holds.

\section{References}

[1] S. Eilenberg, D. Montgomery, Fixed point results for multi-valued transformations, Amer. J. Math., 68 (1946), $214-222$. 1

[2] R. Engelking, General Topology, PWN-Polish Scientific Publishers, Warszawa, (1989). 2

[3] P. M. Fitzpartrick, W. V. Petryshyn, Fixed point theorems for multivalued noncompact acyclic mappings, Pac. J. Math., 54 (1974), 12-23. 1

[4] M. Furi, P. Pera, A continuation method on locally convex spaces and applications to ordinary differential equations on noncompact intervals, Ann. Pol. Math., 47 (1987), 331-346. 1

[5] L. Gorniewicz, Topological fixed point theory of multivalued mappings, Kluwer Academic Publishers, Dordrecht, (1999). 1, 1

[6] A. Granas, Sur la méthode de continuité de Poincaré, C. R. Acad. Sci. Paris Sér. A-B, 282 (1976), 983-985. 1

[7] A. Granas, J. Dugundji, Fixed Point Theory, Springer-Verlag, New York, (2003). 1

[8] D. O'Regan, Fixed point theory on extension type spaces on topological spaces, Fixed Point Theory and Applications, 1 (2004), 13-20. 1, 1

[9] D. O'Regan, Some general theorems for compact acyclic maps, Mathematics, 7 (2019), 11 pages. 1

[10] D. O'Regan, A topological coincidence theory for multifunctions via homotopy, Mathematics, 8 (2020), 8 pages. 1 